\title{
RESPOSTA DO ABACAXIZEIRO 'VITÓRIA' A DOSES DE NITROGÊNIO EM SOLOS DE TABULEIROS COSTEIROS DA PARAÍBA ${ }^{(1)}$
}

\author{
André Luiz Pereira da Silva( ${ }^{(2)}$, Alexandre Paiva da Silva ${ }^{(3)}$, Adailson \\ Pereira de Souza ${ }^{(4)}$, Djail Santos ${ }^{(4)}$, Silvanda de Melo Silva ${ }^{(4)} \&$ Valéria \\ Borges da Silva ${ }^{(5)}$
}

\begin{abstract}
RESUMO
Apesar de importantes, são bastante escassas as informações sobre adubação e nutrição mineral de cultivares de abacaxi resistentes à fusariose. $O$ objetivo deste trabalho foi avaliar a resposta do abacaxizeiro 'Vitória', em termos de nutrição mineral, produção e qualidade, à aplicação de doses crescentes de $\mathrm{N}$, em Espodossolo Humilúvico da região de Tabuleiros Costeiros, Estado da Paraíba. O experimento foi conduzido em delineamento experimental de blocos casualizados, com cinco tratamentos, correspondente às doses de 100, 200, 300, $500 \mathrm{e} 600 \mathrm{~kg} \mathrm{ha}^{-1}$ de $\mathrm{N}$ (ureia aos 60, 180 e 270 dias após o plantio), e três repetições. Avaliaram-se o comprimento, a matéria fresca da folha ' $\mathrm{D}$ ' e os teores de N, P e K nas porções clorofilada e aclorofilada aos 300 e 420 dap; e peso médio, produtividade, atributos físicos (peso, comprimento e diâmetro mediano de infrutescências; peso e comprimento de coroa) e químicos ( $\mathrm{pH}$, sólidos solúveis - SS, acidez titulável - AT e relação SS/AT) de infrutescências na colheita. A elevação da dose de N aumentou linearmente os valores de comprimento e matéria fresca da folha ' $\mathrm{D}$ '. $\mathrm{O}$ aumento das doses promoveu também elevação dos teores de $\mathrm{N}$ e diminuição dos de $\mathrm{P}$ e K da porção clorofilada aos 300 dap, sem alterar, entretanto, os teores desses nutrientes aos 420 dap. Na porção aclorofilada, a elevação das doses de $\mathrm{N}$ aumentou os teores de $\mathrm{K}$ aos 320 dap, mas diminuiu-os aos 420 dap. Os valores de peso de infrutescências e de produtividade aumentaram com a elevação
\end{abstract}

(1) Parte da Dissertação de Mestrado apresentada pelo primeiro autor ao Programa de Pós-Graduação em Manejo de Solo e Água (PPGMSA), da Universidade Federal da Paraíba - UFPB. Recebido para publicação em 20 de janeiro de 2011 e aprovado em 9 de janeiro de 2012.

(2) Doutorando do Programa de Pós-Graduação em Ciência do Solo, Universidade Estadual Paulista - FCAV/UNESP. CEP 14883-354 Jaboticabal (SP). E-mail: andreengagronomo@gmail.com

(3) Professor Adjunto, Centro de Ciências e Tecnologia Agroalimentar (CCTA), Universidade Federal de Campina Grande UFCG. Rua Jairo Vieira Feitosa, s/n, Bairro dos Pereiros, CEP 58840-000 Pombal (PB). E-mail: paivadasilva@gmail.com

(4) Professor Adjunto, Centro de Ciências Agrárias (CCA), Universidade Federal da Paraíba - UFPB. CEP 58397-000 Areia (PB). E-mails: adailson@cca.ufpb.br; santosdj@cca.ufpb.br; silvandasilva@gmail.com

(5) Mestranda do PPGMSA/CCA, UFPB. E-mail: valeria_borgess@hotmail.com 


\begin{abstract}
das doses de $\mathrm{N}$, estimando-se valores máximos de $1,0 \mathrm{~kg}$ e $37,9 \mathrm{t} \mathrm{ha}^{-1}$, com doses de 409,0 e 439,0 $\mathrm{kg} \mathrm{ha}^{-1}$ de $\mathrm{N}$, respectivamente. As doses de $\mathrm{N}$ não alteraram os atributos de qualidade das infrutescências, as quais se mostraram compatíveis com as características descritas para a cultivar.
\end{abstract}

Termos de indexação: Ananas comosus comosus L., adubação nitrogenada, fusariose.

\title{
SUMMARY: RESPONSE OF 'VITÓRIA' PINEAPPLE TO NITROGEN IN COASTAL TABLELANDS IN PARAIBA
}

\begin{abstract}
Informations about fertilization and mineral nutrition of the new fusariosis-resistant pineapple cultivars are still scarce, despite the importance of this crop. The purpose of this study was to evaluate the effect of increasing doses of $N$ on fruit yield and quality of the pineapple cultivar 'Vitória' grown on a 'Humilúvico Espodossolo' in the region of Tabuleiros Costeiros, in the state of Paraiba. The experiment was arranged in a randomized block design, using five $N$ doses (100, 200, 300, 500 and $600 \mathrm{~kg} \mathrm{ha}^{-1} \mathrm{~N}$, as urea) applied 60, 180 and 270 days after planting (dap) with three replications. The following variables were evaluated: length and fresh matter of the ' $D$ ' leaf; contents of $N, P$ and $K$ in the portions with and without chlorophyll, 300 and 420 dap; average weight, yield, physical properties (fruit weight, length and medium diameter; crown weight and length) and chemical properties ( $p H$, soluble solids -SS, titrable acidity-AT, and the SS/AT ratio) of the fruits at harvest. Increasing $N$ doses linearly increased length and fresh matter of the ' $D$ ' leaf and $N$ content and decrease both $P$ and $K$ contents of the chlorophyll portion 300 dap, but did not affect nutrient contents 420 dap; in the chlorophyll-free portion. Increasing $N$ doses, also, led to an increase in $K$ contents 320 dap, but to reductions 420 dap. Individual fruit weight and total yield increased with increasing $N$ doses, as maximum values of $1.0 \mathrm{~kg}$ and $37.9 \mathrm{tha}$ were estimated at doses of 409.0 and $439.0 \mathrm{~kg} \mathrm{ha}^{-1} \mathrm{~N}$, respectively. The $N$ doses did not affect the fruit quality properties, which were compatible with the characteristics described for this cultivar.
\end{abstract}

Index terms: Ananas comosus comosus L., nitrogenous fertilization, fusariosis.

\section{INTRODUÇÃO}

O abacaxizeiro é a terceira fruteira tropical mais cultivada no mundo, sendo o Brasil um dos maiores produtores mundiais, com produção estimada em 1,47 bilhão de frutos numa área colhida de 60,2 mil ha (IBGE, 2010). No País, o Estado da Paraíba destaca-se como principal produtor, tendo produzido, em 2009, cerca de 270 milhões de frutos, o que corresponde a $18 \%$ da produção nacional (IBGE, 2010).

A região abacaxicultora do Estado da Paraíba está concentrada nas microrregiões litorâneas, em áreas de Tabuleiros Costeiros, nas quais predominam solos de textura arenosa, ácidos e de baixa fertilidade natural (Oliveira et al., 2002; Souza et al., 2007; Rodrigues et al., 2010). A produção é feita basicamente por pequenos produtores (áreas $<5$ ha), sem irrigação, utilizando-se a cultivar Pérola (Oliveira et al., 2002; Souza et al., 2007; Silva et al., 2009; Rodrigues et al., 2010). Contudo, tem-se observado a adoção de novas tecnologias e a introdução de novas cultivares, visando atender à demanda de novos nichos de mercado (Souza et al., 2007; Rodrigues et al., 2010).

No Brasil, a abacaxicultura baseia-se apenas nas cultivares Pérola, destinada ao consumo in natura, e Smooth Cayenne, destinada principalmente à agroindústria, as quais têm o inconveniente de serem suscetíveis à fusariose, principal problema fitossanitário da cultura no País (Reinhardt et al., 2002; Ventura et al., 2009). O uso de cultivares resistentes é uma das alternativas para contornar o problema da fusariose (Reinhardt et al., 2002; Ventura et al., 2009), sendo a cultivar 'Vitória' considerada resistente e de dupla aptidão (consumo in natura e agroindústria) (Ventura et al., 2009). No entanto, por ser recente, as informações sobre as exigências nutricionais e a resposta às doses de nutrientes para essa cultivar ainda são incipientes (Baldotto et al., 2009).

O N é o nutriente responsável pelo aumento da produtividade do abacaxizeiro, e a sua deficiência altera negativamente o crescimento, a produção e 
a qualidade das infrutescências (Rao et al., 1977; Mustaffa, 1988; Bhugaloo, 1998; Veloso et al., 2001; Teixeira et al., 2002; Malézieux \& Bartholomew, 2003; Spironello et al., 2004; Silva et al., 2009). O $\mathrm{N}$ desempenha funções importantes no metabolismo ácido das crassuláceas (CAM), além de integrar uma série de compostos indispensáveis ao crescimento e desenvolvimento do abacaxizeiro, incluindo bases nitrogenadas de nucleotídeos e aminoácidos, proteínas, moléculas de clorofila, entre outros (Malézieux \& Bartholomew, 2003; Malavolta, 2006; Vieira et al., 2010).

Diversos trabalhos relatam efeitos positivos do aumento das doses de $\mathrm{N}$ sobre o crescimento e a produção do abacaxizeiro de diferentes cultivares (Nogueira et al., 1970; Lacoeuilhe, 1971; Rao et al., 1977; Choairy \& Fernandes, 1981; Asoegwu, 1987; Mustaffa, 1988; Bhugaloo, 1998; Souza, 2000; Teixeira et al., 2002; Veloso et al., 2002; Spironello et al., 2004; Silva, 2006). Contudo, as doses recomendadas são bastante variáveis (entre 6 e 10 g/planta), o que se deve aos diversos fatores envolvidos na resposta da cultura ao nutriente (densidade de plantio, produtividade esperada, condições edafoclimáticas, práticas de manejo, forma de comercialização e cultivar, entre outros) (Souza, 2000; Malézieux \& Bartholomew, 2003; Silva et al., 2009).

$\mathrm{Na}$ Paraíba, as informações de pesquisa são mais escassas e limitam-se, em sua maioria, à cultivar Pérola (Nogueira et al., 1970; Choairy \& Fernandes, 1981; Silva, 2006; Silva et al., 2009). As recomendações não contemplam a variação do fator produtividade esperada, sendo prescritas doses fixas de $300 \mathrm{~kg} \mathrm{ha}^{-1}$ de $\mathrm{N}$, parceladas equitativamente aos 60, 180 e 270 dias após o plantio (dap) (Emater, 1979; Silva et al., 2009). No entanto, Silva (2006) observou que, para maximizar a produção da cultivar Pérola
(53,3 t ha ${ }^{-1}$ ), foram necessários 401,3 $\mathrm{kg} \mathrm{ha}^{-1}$ de N, justificando assim a realização de trabalhos para condições edafoclimáticas específicas, inclusive para cultivares resistentes à fusariose.

Pelo exposto, e partindo-se do pressuposto de que as doses e os esquemas de parcelamento adotados para a cultivar Pérola são adequados para outras cultivares, este trabalho objetivou avaliar a resposta do abacaxizeiro 'Vitória' - em termos de crescimento vegetativo, nutrição mineral, produção e qualidade dos frutos - à aplicação de doses crescentes de N, em solos de Tabuleiros Costeiros do Estado da Paraíba.

\section{MATERIAL E MÉTODOS}

O trabalho foi realizado na Fazenda Sertaneja, município de Santa Rita, região de Tabuleiros Costeiros do Estado da Paraíba, a qual está definida pelas coordenadas geográficas de $7^{\circ} 14^{\prime} 22^{\prime}$ S, $34^{\circ} 59^{\prime} 13^{\prime \prime} \mathrm{W}$ e altitude de $16 \mathrm{~m}$. O clima predominante é do tipo As' (quente e úmido), com temperatura média entre 22 e $26{ }^{\circ} \mathrm{C}$ e precipitação pluvial de $1.500 \mathrm{~mm}$, concentrados nos meses de fevereiro a junho (Brasil, 1972). O solo da área experimental está classificado como Espodossolo Humilúvico (Embrapa, 2006), e seus atributos físicos (Embrapa, 1997) e químicos (Tedesco et al., 1995) na camada de 0-20 cm, antes da instalação do experimento, são apresentados no quadro 1.

$\mathrm{O}$ experimento foi conduzido em delineamento experimental de blocos casualizados com cinco tratamentos e três repetições, totalizando 15 unidades experimentais. Os tratamentos constaram de cinco doses de $\mathrm{N}\left(100,200,300,500\right.$ e $600 \mathrm{~kg} \mathrm{ha}^{-1}$ de $\mathrm{N}$ ), e a unidade experimental constou de uma

Quadro 1. Atributos químicos e físicos do solo da área experimental, na camada de 0-20 cm, antes da instalação do experimento

\begin{tabular}{|c|c|c|c|}
\hline Atributo químico & Valor & Atributo físico & Valor \\
\hline pH em água $1: 2,5$ & 4,8 & Areia $\left(\mathrm{g} \mathrm{kg}^{-1}\right)$ & 904,0 \\
\hline $\mathrm{MO}\left(\mathrm{g} \mathrm{dm}^{-3}\right)$ & 23,8 & Silte $\left(\mathrm{g} \mathrm{kg}^{-1}\right)$ & 72,0 \\
\hline $\mathrm{P}\left(\mathrm{g} \mathrm{dm}^{-3}\right)$ & 23,9 & Argila $\left(\mathrm{g} \mathrm{kg}^{-1}\right)$ & 24,0 \\
\hline $\mathrm{K}^{+}\left(\mathrm{cmol}_{\mathrm{c}} \mathrm{dm}^{-3}\right)$ & 0,09 & ADA $\left(\mathrm{g} \mathrm{kg}^{-1}\right)$ & 13,0 \\
\hline $\mathrm{Ca}^{2+}\left(\mathrm{cmol}_{\mathrm{c}} \mathrm{dm}^{-3}\right)$ & 2,65 & $\mathrm{GF}\left(\mathrm{g} \mathrm{kg}^{-1}\right)$ & 45,8 \\
\hline $\mathrm{Mg}^{2+}\left(\mathrm{cmol}_{\mathrm{c}} \mathrm{dm}^{-3}\right)$ & 0,53 & $\mathrm{Ds}\left(\mathrm{g} \mathrm{cm}^{-3}\right)$ & 1,38 \\
\hline $\mathrm{SB}\left(\mathrm{cmol}_{\mathrm{c}} \mathrm{dm}^{-3}\right)$ & 3,35 & $\mathrm{Dp}\left(\mathrm{g} \mathrm{cm}^{-3}\right)$ & 2,63 \\
\hline $\mathrm{Na}^{+}\left(\mathrm{cmol}_{\mathrm{c}} \mathrm{dm}^{-3}\right)$ & 0,08 & $\operatorname{Pt}\left(\mathrm{m}^{3} \mathrm{~m}^{-3}\right)$ & 0,47 \\
\hline $\mathrm{H}+\mathrm{Al}\left(\mathrm{cmol}_{\mathrm{c}} \mathrm{dm}^{-3}\right)$ & 10,7 & Classe textural & Areia \\
\hline $\mathrm{Al}^{3+}\left(\mathrm{cmol}_{\mathrm{c}} \mathrm{dm}^{-3}\right)$ & 0,41 & & \\
\hline $\mathrm{CTC}_{\text {efetiva }}\left(\mathrm{cmol}_{\mathrm{c}} \mathrm{dm}^{-3}\right)$ & 3,76 & & \\
\hline $\mathrm{CTC}_{\text {total }}\left(\mathrm{cmol}_{\mathrm{c}} \mathrm{dm}^{-3}\right)$ & 14,1 & & \\
\hline $\mathrm{V}(\%)$ & 24,0 & & \\
\hline
\end{tabular}

MO: matéria orgânica; SB: soma de bases $\left(\mathrm{Ca}^{2+}+\mathrm{Mg}^{2+}+\mathrm{K}^{+}+\mathrm{Na}^{+}\right)$; $\mathrm{CTC}_{\text {total }}$ : $\mathrm{SB}+\mathrm{Al}^{3+}$; CTC efetiva: $\mathrm{SB}+(\mathrm{H}+\mathrm{Al})$; V: saturação por bases: $\left(\mathrm{SB} / \mathrm{CTC}_{\text {total }}\right) \times 100$; $\mathrm{ADA}$ : argila dispersa em água; GF: grau de floculação; Ds: densidade do solo; Dp: densidade de partícula; Pt: porosidade total. 
parcela, contendo 20 plantas de abacaxizeiro, dispostas em sistema de fileiras duplas, no espaçamento de $0,90 \times 0,40 \times 0,30 \mathrm{~m}$. Foram utilizadas mudas micropropagadas do abacaxizeiro 'Vitória', aclimatadas e padronizadas quanto ao tamanho e peso (20 cm e $200 \mathrm{~g}$, respectivamente). As mudas foram provenientes do Centro Nacional de Pesquisa de Mandioca e Fruticultura (EmbrapaCNPMF), sediado em Cruz das Almas, Bahia.

O plantio das mudas foi efetuado em abril de 2007, após as operações de preparo do solo, que constaram de duas gradagens cruzadas e incorporação dos restos culturais do abacaxizeiro 'Pérola' do ciclo anterior.

As doses de $\mathrm{N}$ foram parceladas em três aplicações (60, 180 e 270 dias após o plantio - dap) e aplicadas no solo, junto à base da planta, utilizandose como fontes a fórmula 16-08-24 aos 60 dap e ureia aos 180 e 270 dap. Além das doses de $\mathrm{N}$, foram aplicados $60 \mathrm{~kg} \mathrm{ha}^{-1}$ de $\mathrm{P}_{2} \mathrm{O}_{5}$ e $500 \mathrm{~kg} \mathrm{ha}^{-1}$ de $\mathrm{K}_{2} \mathrm{O}$. A dose de $\mathrm{P}$ foi aplicada aos 60 dap, utilizando-se a fórmula 16-08-24, ao passo que as doses de $\mathrm{K}$ foram parceladas em três aplicações (60, 180 e 270 dap), utilizando-se a fórmula 16-08-24 aos 60 dap e cloreto de potássio aos 180 e 270 dap. As plantas receberam ainda pulverizações foliares de micronutrientes (B, Cu, Fe, Mn e Zn) aos 120, 180, 240 e 300 dap, utilizando-se como fontes bórax, sulfato de cobre, sulfato de ferro, sulfato de manganês e sulfato de zinco, respectivamente (Oliveira et al., 2002).

O experimento foi conduzido em condições de sequeiro, com irrigação suplementar por meio de aspersão, nos meses de menor precipitação (setembro a dezembro). Durante o experimento foram realizadas as práticas culturais recomendadas para a cultura (capinas e controle preventivo de pragas e doenças), visando assegurar condições adequadas de limpeza e sanidade (Oliveira et al., 2002). As plantas não apresentaram quaisquer sintomas de ataque de cochonilha e de fusariose, razão pela qual foram dispensadas as aplicações de defensivos (fungicidas e inseticidas). A indução floral foi feita aos 420 dap, aplicando-se $50 \mathrm{~mL}$ de solução de carbureto de cálcio a $1 \%$, no centro da roseta foliar de cada planta (Oliveira et al., 2002).

Para avaliar o efeito das doses de $\mathrm{N}$ sobre o crescimento vegetativo, foram coletadas cinco folhas 'D' em cada unidade experimental, aos 300 dap e na época de indução floral (420 dap). Nas referidas amostras, foram determinados o comprimento e matéria fresca da folha ' $D$ '.

$\mathrm{O}$ estado nutricional das plantas foi avaliado a partir da amostragem de cinco folhas ' $\mathrm{D}$ ', em cada unidade experimental, aos 300 e 420 dap. Após coletadas, as amostras foram lavadas, secas em estufa $\left(65^{\circ} \mathrm{C}\right.$ por $\left.72 \mathrm{~h}\right)$ e passadas em moinho tipo Wiley; nas subamostras, foram determinados os teores de $\mathrm{N}, \mathrm{P}$ e $\mathrm{K}$ nas porções clorofiladas e aclorofiladas (Tedesco et al., 1995).

A colheita foi realizada aos 600 dap, colhendo-se todas as infrutescências da unidade experimental. Em seguida, elas foram pesadas e categorizadas nas classes comerciais da cultivar Pérola, propostas por Almeida et al. (2004): infrutescências da classe I (peso inferior a 1,20 kg); infrutescências da classe II (peso entre 1,20 e 1,50 kg); infrutescências da classe III (peso entre 1,50 e 1,80 kg); e infrutescências da classe IV (peso superior a $1,80 \mathrm{~kg}$ ). A produtividade foi estimada considerando o percentual de infrutescências de cada classe comercial e um estande final de 36.000 plantas ha ${ }^{-1}$. Das infrutescências colhidas, foram retiradas subamostras para determinar os atributos físicos (peso, comprimento e diâmetro mediano de infrutescências; peso e comprimento da coroa) e químicos $(\mathrm{pH}$, sólidos solúveis - SS), acidez titulável - AT e relação SS/AT), conforme procedimentos descritos em AOAC (1992).

Os dados foram submetidos às análises de variância e de regressão, sendo adotados como critérios para escolha dos modelos de regressão o significado biológico, a significância dos estimadores dos parâmetros de regressão até $10 \%$ e os valores de $\mathrm{R}^{2}$ (Steel et al., 1997).

\section{RESULTADOS E DISCUSSÃO}

A elevação das doses de $\mathrm{N}$ aumentou linearmente os valores de comprimento e de peso da folha ' $D$ ' de abacaxizeiros 'Vitória' nas amostragens feitas aos 300 e 420 dap (Figura 1). Em relação ao comprimento, registraram-se aos 300 dap valores de $69,7 \mathrm{~cm}$ na menor (100 kg ha ${ }^{-1}$ de N) e de 70,9 cm na maior dose $\left(600 \mathrm{~kg} \mathrm{ha}^{-1}\right.$ de $\left.\mathrm{N}\right)$; aos $420 \mathrm{dap}$, foram observados valores de $77,6 \mathrm{~cm}$ na dose de $100 \mathrm{~kg} \mathrm{ha}^{-1}$ e de $81,4 \mathrm{~cm}$ na dose de $600 \mathrm{~kg} \mathrm{ha}^{-1} \mathrm{de}$ $\mathrm{N}$, o que representou incrementos de 11 e $13 \%$, respectivamente (Figura 1).

Quanto ao peso da folha ' $\mathrm{D}$ ', foram registrados aos 300 dap valores de $30,8 \mathrm{~g}$ para a dose de $100 \mathrm{~kg} \mathrm{ha}^{-1}$ e de $41,7 \mathrm{~g}$ para a dose de $600 \mathrm{~kg} \mathrm{ha}^{-1}$ de $\mathrm{N}$, o que equivale a uma diferença de $26 \%$. Aos 420 dap foram registrados valores de $35,9 \mathrm{~g}$ na dose de $100 \mathrm{~kg} \mathrm{ha}^{-1}$ e de $47,1 \mathrm{~g}$ na dose de $600 \mathrm{~kg} \mathrm{ha}^{-1}$ de N, ou seja, um incremento de $24 \%$ (Figura 1).

Aumentos nos valores de comprimento e de peso da folha ' $D$ ' de diferentes cultivares de abacaxizeiros, em função da elevação das doses de $\mathrm{N}$, foram reportados por diversos autores (Lacoeuilhe, 1971; Rao et al., 1977; Singh et al., 1977; Asoegwu, 1987; Mustaffa, 1988), variando, no entanto, a magnitude dos efeitos e os valores das respectivas variáveis. Bhugaloo (1998), entretanto, não constatou efeitos das doses de $\mathrm{N}$ sobre o peso e registrou tendência de 

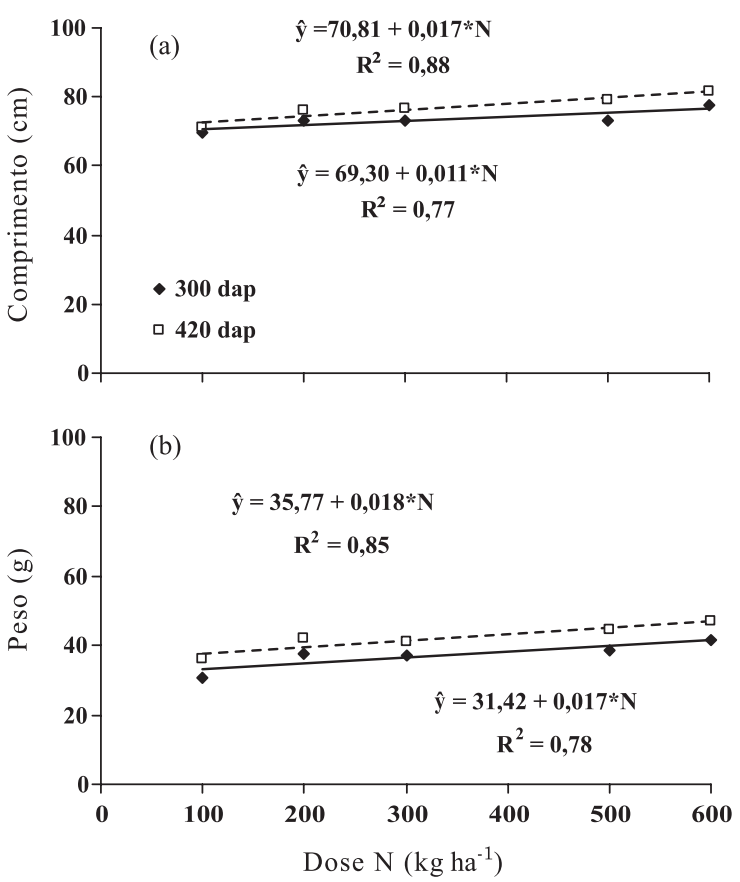

Figura 1. Comprimento (a) e peso da folha ' $D$ ' (b) de abacaxizeiros 'Vitória', aos 300 e 420 dias após o plantio (dap), em função das doses de N. "significativo a $5 \%$ pelo teste $F$.

redução no comprimento da folha 'D' de abacaxizeiros 'Queen Victoria' com a elevação das doses de N (entre 0 e $840 \mathrm{~kg} \mathrm{ha}^{-1}$ na forma de ureia). Ressalta-se que no trabalho conduzido por Bhugaloo (1998) as doses foram aplicadas via fertirrigação e parceladas em sete aplicações, o que aumentaria a eficiência de aproveitamento pelas plantas do nutriente aplicado via fertilizante (Silva et al., 2009).

$\mathrm{O}$ suprimento adequado de $\mathrm{N}$, conforme a demanda do abacaxizeiro, em seus diferentes estádios fenológicos, torna-se essencial para manter altas taxas de crescimento vegetativo e obter altas produtividades (Lacoeuilhe, 1971; Malézieux \& Bartholomew, 2003; Rodrigues et al., 2010). Isso se explica pelo fato de o $\mathrm{N}$ participar da constituição de aminoácidos e proteínas, compostos essenciais para o processo de crescimento vegetal, além de ser indispensável para a utilização de carboidratos no interior da planta, contribuindo para aumentar o número, o peso unitário das folhas, o vigor e o peso total da planta - características estas que se correlacionam positivamente com o peso médio das infrutescências e a produtividade (Reinhardt et al.,1987; Hepton, 2003; Hanafi \& Halimah, 2004).

Nas condições em que não ocorram estresses durante a formação da infrutescência, estimase que plantas da cultivar Smooth Cayenne, parental da cultivar 'Vitória', que apresentem na época de indução floral valores de peso e de comprimento da folha 'D' em torno de $100 \mathrm{~g}$ e de $70 \mathrm{~cm}$, respectivamente, sejam capazes de produzir infrutescências com peso superior a $1,5 \mathrm{~kg}$ (Reinhardt et al.,1987). Neste trabalho, mesmo sem ter havido estresses, os valores de peso (entre 35,9 e $47,1 \mathrm{~g}$ ) e de comprimento da folha 'D' (entre 77,6 e $81,4 \mathrm{~cm}$ ) na época de indução floral ficaram abaixo desses valores de referência (Figura 1), o que pode ser justificado pelo fato de terem sido utilizadas mudas meristemáticas (Reinhardt et al.,1987; Baldotto et al., 2009).

Constata-se assim que, mesmo tendo sido usadas mudas de tamanho ( $20 \mathrm{~cm}$ de altura) e peso adequados (aproximadamente $200 \mathrm{~g}$ ) e observados os períodos de aclimatização (em casa de vegetação) e de aclimatação (em canteiros) (Berilli et al., 2011), o crescimento das plantas foi lento, o que se deveu à baixa capacidade de aproveitamento das doses de $\mathrm{N}$ aplicadas, refletindo, portanto, em baixos valores de peso e comprimento da folha ' $\mathrm{D}$ ' tanto aos 300 quanto aos 420 dap (Figura 1).

Com relação ao estado nutricional das plantas, observou-se que aos 300 dap a elevação das doses de $\mathrm{N}$ aumentou os teores de $\mathrm{N}$ da porção clorofilada, mas não influenciou os da porção aclorofilada (Figura 2a). Pela equação de regressão ajustada, estimou-se teor máximo de $15,1 \mathrm{~g} \mathrm{~kg}^{-1}$ com a dose de $405,8 \mathrm{~kg} \mathrm{ha}^{-1}$ de $\mathrm{N}$ para a porção clorofilada e teor médio de $9,4 \mathrm{~g} \mathrm{~kg}^{-1}$ de $\mathrm{N}$ para a porção aclorofilada (Figura 2a). Aos 420 dap, ou seja, na época de indução floral, os dados não se ajustaram aos modelos de regressão testados, estimando-se teores médios de $7,3 \mathrm{~g} \mathrm{~kg}^{-1}$ para a porção clorofilada e de $7,5 \mathrm{~g} \mathrm{~kg}^{-1}$ para a aclorofilada (Figura $2 \mathrm{~b}$ ).

A elevação das doses de $\mathrm{N}$ diminuiu os teores de $\mathrm{P}$ da porção clorofilada aos 300 dap, porém não influenciou os da aclorofilada (Figura 2c). Pela equação de regressão ajustada, estimou-se teor mínimo de $1,8 \mathrm{~g} \mathrm{~kg}^{-1}$ na dose de $600 \mathrm{~kg} \mathrm{ha}^{-1}$ de N para a porção clorofilada e teor médio de $3,4 \mathrm{~g} \mathrm{~kg}^{-1}$ de $\mathrm{N}$ para a porção aclorofilada (Figura 2c). Aos 420 dap, estimaram-se teores médios de 2,0 e $2,4 \mathrm{~g} \mathrm{~kg}^{-1}$ para as porções clorofilada e aclorofilada, respectivamente (Figura $2 \mathrm{~d}$ ).

Quanto aos teores de K, observou-se que o aumento das doses de $\mathrm{N}$ reduziu os teores da porção clorofilada e aumentou os da aclorofilada aos 300 dap (Figura 2e). Pelas equações de regressão ajustadas, estimou-se teor mínimo de $24,9 \mathrm{~g} \mathrm{~kg}^{-1}$ na maior dose de $\mathrm{N}$ para a porção clorofilada e teor máximo de $39,1 \mathrm{~g} \mathrm{~kg}^{-1}$ na dose de 443,0 $\mathrm{kg} \mathrm{ha}^{-1}$ de $\mathrm{N}$ para a porção aclorofilada (Figura 2e). Aos 420 dap, observou-se que as doses de $\mathrm{N}$ não influenciaram os teores da porção clorofilada, mas diminuíram os da aclorofilada (Figura 2f), estimando-se teor médio de 19,3 $\mathrm{g} \mathrm{kg}^{-1}$ e teor mínimo de $16,3 \mathrm{~g} \mathrm{~kg}^{-1}$, respectivamente (Figura $2 \mathrm{f}$ ). 

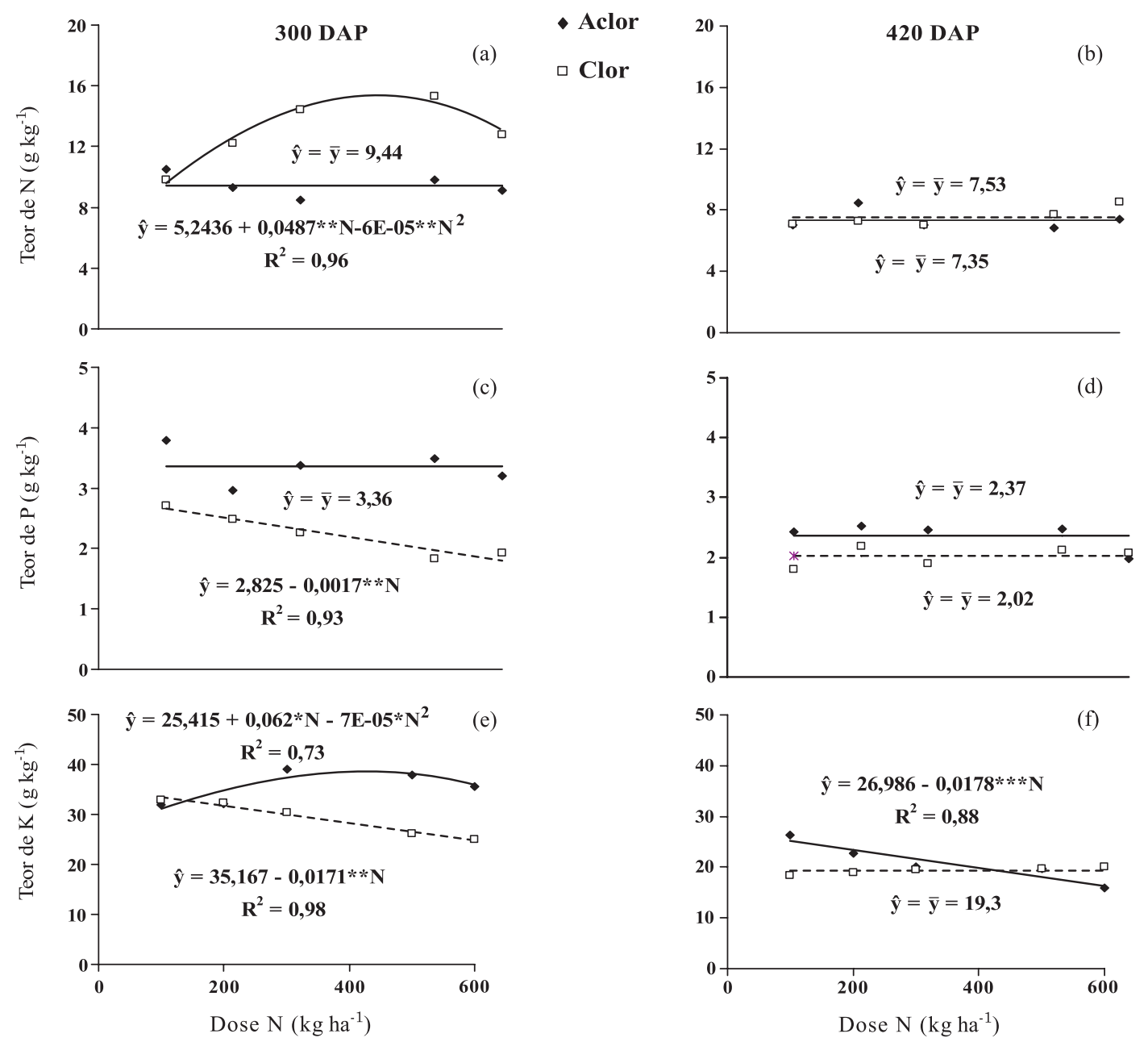

Figura 2. Teores de N (A e B), P (C e D), e K (D e F) em folhas de abacaxizeiro 'Vitória', nas porções aclorofiladas e clorofiladas, aos 300 e 420 dias após o plantio (dap), em função das doses de $\mathrm{N}^{*}{ }^{* * * *}, \mathrm{e}^{* *}$ significativo a $0,1,1$ e $5 \%$, respectivamente, pelo teste $F$.

Considerando a faixa adequada de teores proposta por Malézieux \& Bartholomew (2003) para o abacaxizeiro 'Smooth Cayenne', observa-se que, com exceção das maiores doses (400 e $500 \mathrm{~kg} \mathrm{ha}^{-1}$ de $\mathrm{N}$ ), aos 300 dap, os teores de $\mathrm{N}$ dos demais tratamentos, tanto aos 300 quanto aos 420 dap, ficaram abaixo da faixa ideal (15 a $\left.17 \mathrm{~g} \mathrm{~kg}^{-1} \mathrm{de} \mathrm{N}\right)$. Quanto aos teores de $\mathrm{P}$, em ambas as amostragens eles se mantiveram acima do teor adequado $\left(1,0 \mathrm{~g} \mathrm{~kg}^{-1}\right)$ (Malézieux \& Bartholomew, 2003), enquanto para K os teores de ambas as porções mantiveram-se dentro da faixa ideal (25,0 a 27,0 $\left.\mathrm{g} \mathrm{kg}^{-1}\right)$ apenas aos 300 dap (Malézieux \& Bartholomew, 2003).

$\mathrm{O}$ aumento dos teores foliares de $\mathrm{N}$ e a redução dos teores de $\mathrm{P}$ e $\mathrm{K}$ da porção clorofilada com a elevação das doses de $\mathrm{N}$ aos 300 dap (Figura 2) está coerente com resultados encontrados por diversos autores (Rao et al., 1977; Asoegwu, 1987; Mustaffa, 1988; Veloso et al., 2001; Teixeira et al., 2002; Spironello et al., 2004), demonstrando a importância do fornecimento de doses adequadas de
$\mathrm{N}$, em função da demanda nutricional, nos diferentes estádios fenológicos (Rodrigues et al., 2010). Por outro lado, a diminuição dos teores de $\mathrm{P}$ e K deveu-se a um possível efeito de diluição dos teores de $\mathrm{N}$ em relação aos de P (Py et al., 1987; Malavolta, 2006; Vieira et al., 2010), bem como ao antagonismo que ocorre entre $\mathrm{N}$ e K no tecido vegetal, respectivamente (Lacoeuilhe, 1971; Py et al., 1987; Spironello et al., 2004; Malavolta, 2006).

Os menores teores de N, P e K aos 420 dap, em ambas as porções, em relação aos observados aos 300 dap (Figura 2), corroboram as afirmações de França (1976) sobre a tendência de diminuição dos teores desses nutrientes na época de indução floral, ocasião em que se inicia a fase de desenvolvimento das infrutescências e na qual ocorre aumento na translocação das reservas nutricionais acumuladas pelas folhas (França, 1976; Mustaffa, 1988; Malézieux \& Bartholomew, 2003). No entanto, devido à maior mobilidade de $\mathrm{N}$ e $\mathrm{K}$ no solo, é provável que a utilização de mudas meristemáticas, a textura 
arenosa do solo e a forma de aplicação das doses (três aplicações no solo e não na axila foliar) tenham favorecido as perdas (lixiviação, principalmente) e diminuído a eficiência de aproveitamento das doses aplicadas desses nutrientes (Teixeira et al., 2002; Malézieux \& Bartholomew, 2003).

No tocante ao efeito da porção da folha analisada, observou-se que, com exceção dos teores de N, foram registrados, apesar da falta de ajuste aos modelos de regressão testados, maiores teores de $\mathrm{P}$ e $\mathrm{K}$ na porção aclorofilada tanto aos 300 quanto aos 420 dap (Figura 2), corroborando assim os resultados de Rao et al. (1977) com a cultivar Kew. Iuchi \& Iuchi (1992) também reportaram maiores teores de P e K na porção aclorofilada da cultivar Smooth Cayenne, porém, diferentemente deste trabalho, registraram maiores teores de $\mathrm{N}$ nessa porção, embora tenha havido correlação mais estreita do peso das infrutescências com os teores da porção clorofilada.

De acordo com Siebeneichler et al. (2002), a porção aclorofilada das folhas do abacaxizeiro normalmente apresenta maiores teores de nutrientes, com exceção de N, S, B, Mn e Fe, em relação às porções clorofilada, apical e na folha inteira, além do fato de que os teores de $\mathrm{N}$ da porção aclorofilada subestimam os teores encontrados na porção clorofilada e na folha inteira. Neste trabalho, os teores de N na porção clorofilada, apesar de inferiores aos da porção aclorofilada aos 300 e 420 dap, apresentaram correlação positiva e significativa com o peso das infrutescências $(\mathrm{r}=$ $0,97^{* *} ; \mathrm{n}=5$; dados não apresentados).

No que se refere à produção, verificou-se que a elevação das doses de $\mathrm{N}$ aumentou tanto o peso de infrutescências quanto a produtividade do abacaxizeiro 'Vitória' (Figura 3). Pelas equações de regressão ajustadas, estimaram-se peso máximo de $1,0 \mathrm{~kg}$ com a dose de $409 \mathrm{~kg} \mathrm{ha}^{-1}$ de $\mathrm{N}$ e produtividade máxima de $37,3 \mathrm{t} \mathrm{ha}^{-1}$ com a dose de $439,0 \mathrm{~kg} \mathrm{ha}^{-1} \mathrm{de}$ N (Figura 4). Respostas positivas de abacaxizeiros de diferentes cultivares à elevação das doses de $\mathrm{N}$

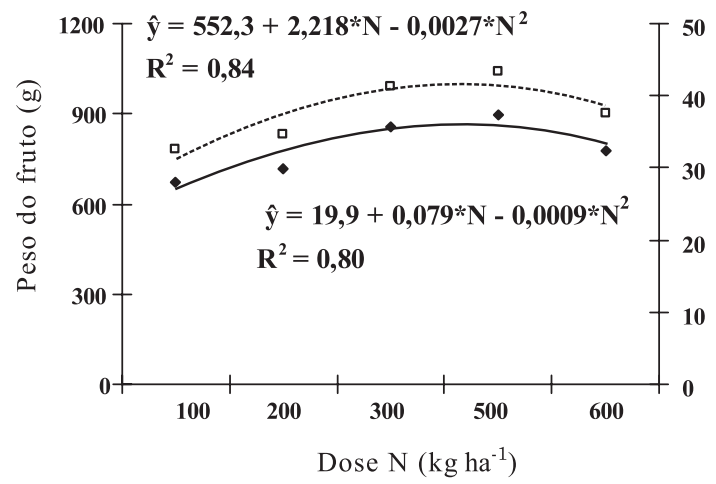

Figura 3. Peso médio de infrutescências ( $($ ) e produtividade (•) de abacaxizeiro 'Vitória', em função das doses de N. "significativo a $5 \%$ pelo teste $F$. sobre o peso das infrutescências e a produtividade foram também relatadas por diversos autores (Rao et al., 1977; Asoegwu, 1987; Bhugaloo, 1998; Mustaffa, 1988; Teixeira et al., 2002; Veloso et al., 2002; Spironello et al., 2004; Silva et al., 2009), variando, contudo, as produtividades e as doses de máxima eficiência física e econômica.

Os efeitos positivos da elevação das doses de $\mathrm{N}$ sobre o peso das infrutescências e a produtividade do abacaxizeiro 'Vitória' observados neste trabalho são explicados pelo aumento dos valores de peso e de comprimento da folha ' $D$ ' (Figura 1) e pela elevação dos teores de N (Figura 2), principalmente na porção clorofilada aos 300 dap $\left(r=0,97^{* *}\right.$; dados não apresentados). Isso corrobora as afirmações de Souza (2000), de que a não aplicação de fertilizantes nitrogenados, em formas orgânicas ou sintéticas, resulta quase sempre no comprometimento dos valores de peso médio e de produtividade, os quais foram baixos neste trabalho (Silva et al., 2009).

A baixa produtividade registrada neste trabalho deveu-se à obtenção de infrutescências com peso médio inferior $(1,0 \mathrm{~kg})$ aos valores de $1,5 \mathrm{~kg}$ reportados por Ventura et al. (2009), em testes preliminares realizados no Espírito Santo. Essas diferenças podem ser atribuídas, além das diferenças nas condições edafoclimáticas entre os dois locais e do manejo das doses de N (forma, época e número de aplicações), ao fato de terem sido utilizadas neste trabalho mudas meristemáticas, as quais apresentaram, devido ao seu caráter juvenil, crescimento lento e baixa capacidade de aproveitamento das doses aplicadas. Isso demonstra que a estratégia de adubação nitrogenada (doses, época e número de aplicações) preconizada para o abacaxizeiro 'Pérola', em solos de Tabuleiros Costeiros Paraibanos, não mostrou-se adequada para o abacaxizeiro 'Vitória', produzido a partir de mudas meristemáticas.

As doses de $\mathrm{N}$ não influenciaram os atributos de qualidade das infrutescências do abacaxizeiro 'Vitória' (Figura 4), uma vez que os dados não se ajustaram aos modelos de regressão testados, estimando-se apenas valores médios (Figura 4). A ausência de efeitos das doses de $\mathrm{N}$ sobre os atributos de qualidade de infrutescências do abacaxizeiro 'Vitória' discorda dos resultados encontrados por Veloso et al. (2002) e Spironello et al. (2004) para as cultivares Pérola e Smooth Cayenne, respectivamente; concorda, entretanto, com Bhugaloo (1998) e Mustaffa (1988), ao não constatarem efeitos consistentes do $\mathrm{N}$ sobre os atributos de qualidade das infrutescências de abacaxizeiros 'Queen Victoria' e 'Smooth Cayenne', respectivamente.

Nitrogênio e potássio são os principais nutrientes que influenciam os atributos de qualidade do 

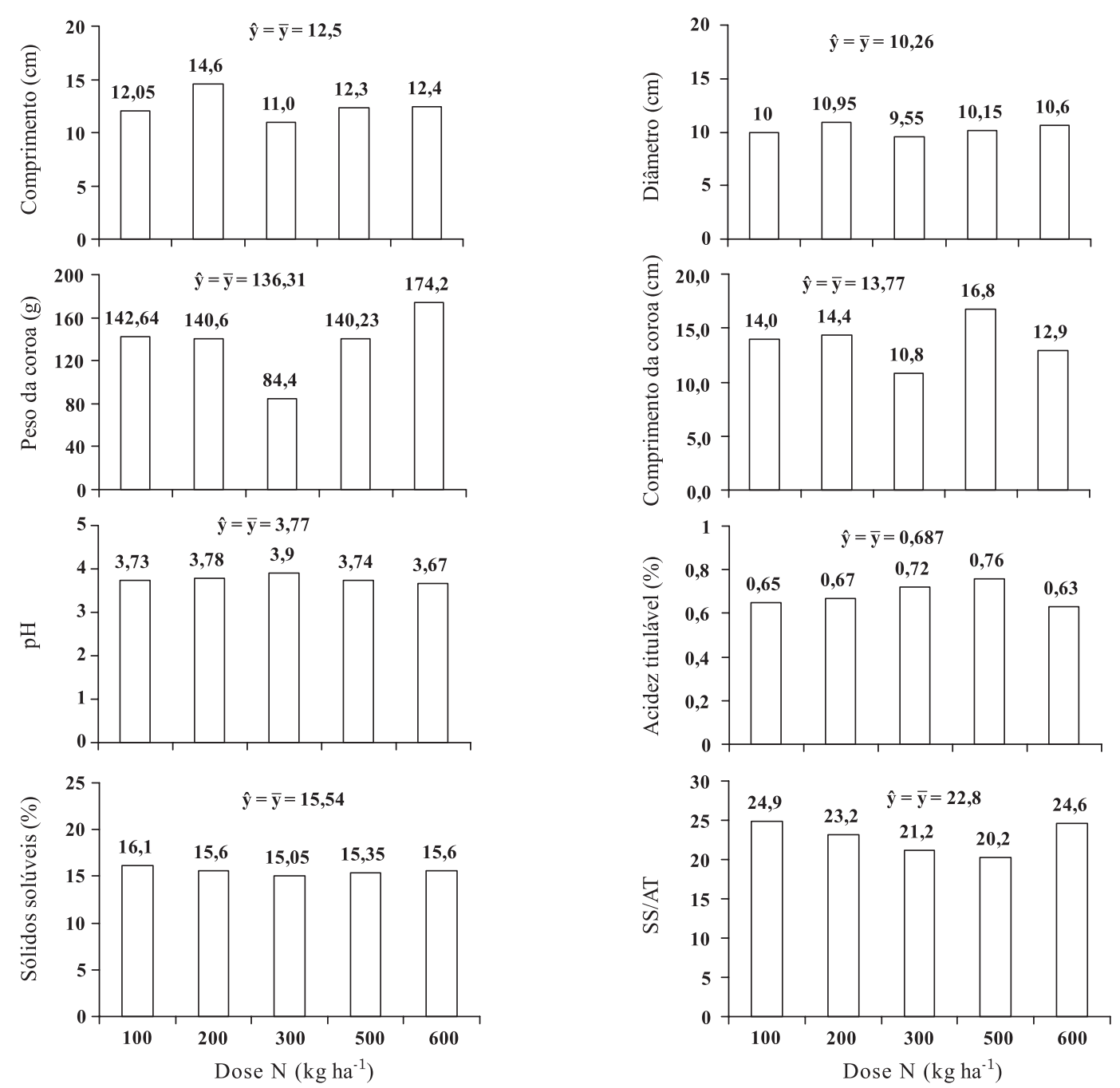

Figura 4. Atributos físicos (comprimento e diâmetro de infrutescências; peso e comprimento de coroa) e químicos (pH, acidez titulável, sólidos solúveis e relação SS/AT) de abacaxizeiros 'Vitória', em função das doses de $\mathbf{N}$.

abacaxizeiro, embora nem sempre seja possível distinguir os efeitos específicos do $\mathrm{N}$ sobre os atributos de qualidade daqueles mais gerais do nutriente sobre o crescimento vegetativo e o vigor das plantas (Py et al., 1987). Em geral, o N aumenta o peso, o tamanho das infrutescências e a espessura da casca, embora em excesso possa reduzir o teor de ácido ascórbico, a consistência e a acidez dos frutos, além de aumentar a translucência da polpa (Malézieux \& Bartholomew, 2003). Além disso, as doses de $\mathrm{N}$ que alteram e, ou, maximizam os atributos de qualidade das infrutescências do abacaxizeiro são, em geral, superiores às doses que maximizam a produtividade (Spironello et al., 2004). Nesse sentido, considerando os baixos valores de peso de infrutescências e de produtividade, em virtude da utilização de mudas meristemáticas, o que comprometeu o aproveitamento das doses, especula-se que os fotoassimilados translocados para as infrutescências (Py et al., 1987; Malézieux \& Bartholomew, 2003) tenham sido destinados ao aumento do peso das infrutescências, em detrimento da melhoria dos atributos de qualidade (Figuras 3 e 4).

Apesar de as doses de $\mathrm{N}$ não terem influenciado os atributos de qualidade das infrutescências do abacaxizeiro 'Vitória' neste trabalho (Figura 4), constatou-se que estes se mostraram compatíveis com as descrições das características das infrutescências desta cultivar, feitas por Ventura et al. (2009), a saber: a) comprimento do fruto $=10,5 \mathrm{vs}$ $12,5 \mathrm{~cm}$ (este trabalho); b) diâmetro central $=12 \mathrm{vs}$ $10,3 \mathrm{~cm}$ (este trabalho); c) comprimento da coroa $=$ $13,8 \mathrm{~cm}$ (este trabalho); d) peso da coroa $=131 \mathrm{vs}$ 136,3 g (este trabalho); e) $\mathrm{pH}=3,8$ (este trabalho); f) acidez titulável $=0,8$ us $0,7 \%$ (este trabalho); g) sólidos solúveis $=15,8$ vs $15,5 \%$ (este trabalho); e g) relação $\mathrm{SS} / \mathrm{AT}=19,8$ vs 22,8 (este trabalho). 


\section{CONCLUSÕES}

Plantas de abacaxizeiro 'Vitória' produzidas a partir de mudas meristemáticas respondem positivamente ao aumento das doses de $\mathrm{N}$ (peso, comprimento e teores de $\mathrm{N}$ na folha ' $\mathrm{D}$ '); contudo, devido ao crescimento vegetativo lento, o peso médio das infrutescências e a produtividade são baixos $\left(1,0 \mathrm{~kg}\right.$ e $\left.37,8 \mathrm{t} \mathrm{ha}^{-1}\right)$ e maximizados com doses de 409,0 e $439,0 \mathrm{~kg} \mathrm{ha}^{-1}$, respectivamente; as doses de $\mathrm{N}$ não alteraram os atributos de qualidade das infrutescências.

\section{AGRADECIMENTOS}

Ao $\mathrm{CNPq}$, pela concessão de auxílio financeiro ao primeiro autor; ao PPGMSA/CCA/UFPB, pelo apoio institucional; ao engenheiro-agrônomo Leôncio da Costa Vilar, pelo apoio técnico; ao senhor José Queiroga, proprietário da Fazenda Sertaneja, pela concessão da área e pelo apoio logístico; aos revisores e editor-chefe da RBCS pelas críticas e sugestões.

\section{LITERATURA CITADA}

ALMEIDA, C.O.; VILAR, L.C.; SOUZA, L.F.S.; REINHARDT, D.H. \& MACEDO, M.C. Peso do abacaxi: Um termo em discussão. Bahia Agríc.,6:1-6, 2004.

ASOEGWU, S.N. Effect of irrigation and nitrogen on the growth and yield of pineapples (Ananas comosus) cv Smooth Cayenne. Fruits, 42:505-509, 1987.

ASSOCATION OF OFFICIAL AGRICULTURAL CHEMIST AOAC. Official methods of analysis of the Association of the Agricultural Chemists. 12.ed. Washington, 1992.

BALDOTTO, L.E.B.; BALDOTTO, M.A.; GIRO, V.B.; CANELLAS, L.P.; OLIVARES, F.L. \& BRESSAN-SMITH, R. Desempenho do abacaxizeiro 'Vitória', em resposta à aplicação de ácidos húmicos durante a aclimatação. R. Bras. Ci. Solo, 33:979-990, 2009.

BERILLI, S.S.; CARVALHO, A.J.C.; FREITAS, S.J.; FARIA, D.C. \& MARINHO, C.C. Avaliação do desenvolvimento de diferentes tamanhos de mudas micropropagadas de abacaxizeiro, após aclimatação. R. Bras.Frutic., 33:208$214,2011$.

BHUGALOO, R.A. Effects of different levels of nitrogen on yield and quality of pineapple variety Queen Victoria. Mauritius, Food Agricultural Research Council, 1998. 80p. (Technical Bulletin)

BRASIL. Ministério da Agricultura. Escritório de Pesquisa e Experimentação. Equipe de Pedologia e Fertilidade do solo. I Levantamento exploratório-reconhecimento de solos do Estado da Paraíba. II Interpretação para uso agrícola dos solos do Estado da Paraíba. Rio de Janeiro, 1972. 638p. (Boletim Técnico, 15; SUDENE, Série Pedológica, 8)
CHOAIRY, S.A. \& FERNANDES, P.D. Adubação NPK em abacaxi (Ananas comosus L, cv. Smooth Cayenne). R. Bras. Frutic., 6:67-76, 1981

EMPRESA BRASILEIRA DE PESQUISA AGROPECUÁRIA . EMBRAPA. Centro Nacional de Pesquisa de Solos. Sistema brasileiro de classificação de solos. 2.ed. Rio de Janeiro, 2006. 399p

EMPRESA BRASILEIRA DE PESQUISA AGROPECUÁRIA EMBRAPA. Centro Nacional de Pesquisa de Solos. Manual de métodos de análise de solos. 2.ed. Rio de Janeiro, 1997. 212p.

EMPRESA DE ASSISTÊNCIA TÉCNICA E EXTENSÃO RURAL DA PARAÍBA - EMATER. Sugestões de adubação para o estado da Paraíba: $1^{a}$ aproximação. João Pessoa, 1979. 105p.

FRANÇA, G.E. Curva de crescimento, concentração e absorção de macronutrientes pelo abacaxizeiro (Ananas comosus L. Merril) durante um ciclo de cultura. Piracicaba, Escola Superior de Agricultura Luiz de Queiroz, 1976. 62p. (Tese de Mestrado)

HANAFI, M.M. \& HALIMAH, A. Nutrient supply and dry-matter partitioning of pineapple cv. Josapine on sandy tin tailings. Fruits, 59:359-366, 2004.

HEPTON, A. Cultural system. In: BARTHOLOMEW, D.P.; PAUL, R.E. \& ROHRBACH, K.G., eds. The Pineapple: Botany, production and uses. Honolulu, CAB, 2003. p.109142.

INSTITUTO BRASILEIRO DE GEOGRAFIA E ESTATÍSTICA IBGE. Levantamento sistemático da produção agrícola. Rio de Janeiro, LSPA, 2009. Disponível em: < http://www.sidra. ibge.gov.br/cgi-bin/prtabl.> Acesso em 20 de dez. de 2010.

IUCHI, V.L. \& IUCHI, T. Escolha da melhor parte da folha 'D' do abacaxizeiro para amostragem de N, P K, Ca e Mg. R. Bras. Frutic., 14:13-20, 1992.

LACOEUILHE, J.J. L'azote et la croissance de l'ananas. Fruits, $26: 37-44,1971$

MALAVOLTA, E. Manual de nutrição mineral de plantas. São Paulo, Ceres, 2006. 638p.

MALÉZIEUX, E. \& BARTHOLOMEW, D.P. Plant nutrition. In: BARTHOLOMEW, D.P.; PAUL, R.E. \& ROHRBACH, K.G., eds. The Pineapple: Botany, production and uses. Honolulu, CAB, 2003. p.143-165.

MUSTAFFA, M.M. Influence of plant population and nitrogen on fruit yield quality and leaf content of Kew pineapple. Fruits, 43:455-458, 1988.

NOGUEIRA, M.A.; LUCAS, A.F.; SILVA, L.G.; SOUZA, L.C. \& SOUZA, I.B. Ensaio de adubação NPK em abacaxi nos Tabuleiros Costeiros do Nordeste. Pesq. Agropec. Nord., $2: 57-71,1970$

OLIVEIRA, E.F.; CARVALHO, R.A.; LACERDA, J.T.; CHOAIRY, S.A. \& BARREIRO NETO, M. Abacaxi: Sistema de cultivo para o tabuleiro paraibano. João Pessoa, EMEPA, 2002. 38p.

PY, C.; LACOEWHILE, J.J. \& TEISSON, C. L' ananás: As culture, sés produts. Paris, G.P. Maisonneuve \& Larose, 1984. $526 \mathrm{p}$ 
RAO, H.; SUBRAMANIANT, T.R.; SRINIVASA MURTHY, H.K.; SINGH, H.P.; DASS, H.C. \& GANAPATHY, K.M. Leaf nitrogen as influenced by varying levels of nitrogen application and its relationship with yield in 'Kew' pineapple. Sci. Hortic., 7:137-142, 1977.

REINHARDT, D.H.; CABRAL, J.R.S.; SOUZA, L.F.S.; SANCHES, N.F. \& MATOS, A.P. Pérola and Smooth Cayenne pineapple cultivars in the state of Bahia, Brazil: Growth, flowering, pests and diseases, yield and fruit quality aspects. Fruits, 57:43-53, 2002.

REINHARDT, D.H.R.C.; COSTA, J.T.A. \& CUNHA, G.A.P. Influência da época de plantio, tamanho da muda e idade da planta para a indução floral do abacaxi Smooth Cayenne no Reconcavo Baiano II - produtividade e características do fruto. Fruits, 42:13-23,1987.

RODRIGUES, A.A.; MENDONÇA, R.M.N.; SILVA, A.P.; SILVA, S.M. \& PEREIRA, W.E. Desenvolvimento vegetativo de abacaxizeiros 'Pérola' e 'Smooth Cayenne' no Estado da Paraíba. R. Bras. Frutic., 32:126-134, 2010.

SIEBENEICHLER, S.C.; MONNERAT, P.H.; CARVALHO, A.J.C. \& SILVA, J.A. Composição mineral da folha em abacaxizeiro: Efeito da parte da folha analisada. R.Bras. Frutic., 24:194-198, 2002.

SILVA, A.P. Sistema de recomendação de fertilizantes e corretivos para a cultura do abacaxizeiro. Viçosa, MG, Universidade Federal de Viçosa, 2006. 176p. (Tese de Doutorado)

SILVA, A.P.; ALVAREZ V.,V. H.; SOUZA, A.P.; NEVES, J.C.L.; NOVAIS, R.F. \& DANTAS, J.P. Sistema de recomendação de fertilizantes e corretivos para a cultura do abacaxizeiro. R. Bras. Ci. Solo, 33:1269-1280, 2009.

SINGH, H.P.; DASS, H.C.; GANAPATHY, K.M. \& SUBRAMANIAN, T.R. Nitrogen requirement of pineapple under irrigated and rainfed conditions. Indian J. Hortic., 34:377-384, 1977.
SOUZA, C.B.; SILVA, B.B. \& AZEVEDO, P.V. Crescimento e rendimento do abacaxizeiro nas condições climáticas dos Tabuleiros Costeiros do Estado da Paraíba. R. Bras. Eng. Agric. Amb., 11:134-141, 2007.

SOUZA, L.F.S. Adubação. In: REINHARDT, D.H.; SOUZA, L.F.S. \& CABRAL, J.R.S., eds. Abacaxi. Produção: Aspectos técnicos. Brasília, Embrapa, 2000. p.30-34.

SPIRONELLO, A.; QUAGGIO, J.A.; TEIXEIRA, L.A.J.; FURLANI, P.R. \& SIGRIST, J.M.M. Pineapple yield and fruit quality effected by NPK fertilization in a tropical soil. R. Bras. Frutic., 26:155-159, 2004.

STEEL, R.G.D.; TORRIE, J.H. \& DICKET, D.A. Principles and procedures of statistics: A biometrical approach. 3.ed. London, McGraw-Hill, 1997. 666p.

TEDESCO, M.J.; GIANELLO, C.; BISSANI, C.A.; BOHNEN, H. \& VOLKWEISS, S.J. Análise de solo, planta e outros materiais. Porto Alegre, Universidade Federal do Rio Grande do Sul, 1995. 174p.

TEIXEIRA, L.A.J.; SPIRONELLO, A.; FURLANI, P.R. \& SIGRIST, J.M.M. Parcelamento da adubação NPK em abacaxizeiro. R. Bras. Frutic., 24:219-224, 2002.

VELOSO, C.A.C.; OEIRAS, A.H.L.; CARVALHO, E.J.M. \& SOUZA, F.R.S. Resposta do abacaxizeiro à adição de nitrogênio, potássio e calcário em Latossolo Amarelo do Nordeste Paraense. R. Bras. Frutic., 23:396-402, 2001.

VENTURA, J.; COSTA, H. \& CAETANO, L.C.S. Abacaxi 'Vitória': Uma cultivar resistente à fusariose. R. Bras. Frutic., 31:931$932,2009$.

VIEIRA, D.P.; PORTES, T.A.; SERAPHIN, E.S. \& TEIXEIRA, J.B. Fluorescência e teores de clorofila em abacaxizeiro cv. Perola submetido a diferentes concentrações de sulfato de amônio. R. Bras. Frutic., 32:360-368, 2010. 